\title{
LA VIOLENCE DIVINE DE BENJAMIN ET LE CAS DE CORÉ (KORAH)
}

La rébellion contre Moïse comme première scène du messianisme

\author{
Petar Bojanic
}

Editions Lignes | Lignes »

2008/3 $n^{\circ} 27 \mid$ pages 125 à 145

ISSN 0988-5226

ISBN 9782355260179

Article disponible en ligne à l'adresse :

https://www.cairn.info/revue-lignes-2008-3-page-125.htm

Distribution électronique Cairn.info pour Editions Lignes.

(C) Editions Lignes. Tous droits réservés pour tous pays.

La reproduction ou représentation de cet article, notamment par photocopie, n'est autorisée que dans les limites des conditions générales d'utilisation du site ou, le cas échéant, des conditions générales de la licence souscrite par votre établissement. Toute autre reproduction ou représentation, en tout ou partie, sous quelque forme et de quelque manière que ce soit, est interdite sauf accord préalable et écrit de l'éditeur, en dehors des cas prévus par la législation en vigueur en France. Il est précisé que son stockage dans une base de données est également interdit. 


\section{La violence divine de Benjamin et le cas de Coré (Korah)}

La rébellion contre Moïse comme première scène du messianisme

\section{Petar Bojanic}

Avant toute chose, pour justifier une lecture supplémentaire du texte de Benjamin Critique de la violence, j'aimerais essayer de présenter tout de suite la manière dont il a été reçu, c'està-dire tellement lu, et expliquer les raisons de ses multiples reconstructions, de Scholem à Löwenthal, jusqu'à Honneth, Žižek ou Butler. Qu'est-ce qui produit la "force déconstructive " de ce dense et complexe collage de différents textes ? Ce n'est pas seulement le brillant montage de Benjamin, mais aussi, me semble-t-il, un puissant malentendu qui dissimule la surprenante analogie du titre de mon texte, entre la violence divine et le nom de Coré. J'aimerais donc immédiatement compléter ce "lien» entre la violence divine et le nom de Coré par deux remarques censées limiter toute interprétation supplémentaire: 1. À la différence de Leo Löwenthal ${ }^{\mathrm{I}}$, j'accepterai de traiter le texte de Benjamin comme une somme de figures et de catégories messianiques et, analogiquement, 2. je suivrai les célèbres qualificatifs que Scholem attribue à ce "texte purement juif» de Benjamin (ein rein jüdischer Text), qu'il conçoit comme une manifestation de "nihilisme positif» ou de "noble et positive violence de destruction" (die edle und positive Gewalt der Zerstörung) ${ }^{2}$. Ces deux éléments (le messianisme et le nihilisme positif) pourraient redoubler mon "intervention "

1.L.Löwenthal, "Gewalt und Recht in der Staats - und Rechtsphilosophie Rousseaus und der deutschen idealistischen Philosophie " (1926, Staatsexamensarbeit), Philosophischen Frühschriften, Frankfurt am Main, Suhrkamp, 1990, S. 174.

2. G. Scholem, "Im Gespräch über Walter Benjamin (1968)", Sinn und Form, 2007, No. 4, S. 501, 502. 
sur le texte de Benjamin, puis peut-être supprimer l'avantage du titre de ce texte sur son sous-titre. Dans ce cas-là, le titre du texte qui évoque le lien entre la "violence divine " et le nom de Coré passerait complètement au second plan. Dominerait alors la géniale suggestion ou intuition de Benjamin que j'ai formulée dans le sous-titre de ce texte : que la première grande rébellion dans l'histoire de la justice (et la dernière dans le cadre du mythe ${ }^{\mathrm{I}}$ ou du droit) évoque ou provoque quelque chose de messianique; que cet épisode majeur dans la vie d'un peuple, dont Coré et quelques-uns des rebelles sont les initiateurs, représente le début de la construction du théâtre messianique.

Mais, inversement, pour autant que l'on essaie de trouver des signes et des formes de messianisme ${ }^{2}$ dans la rébellion, pour autant que, par exemple, Coré - «contrairement à » Benjamin, mais toujours "avec lui "- est bien le "premier contestataire de gauche de toute l'histoire politique ${ }^{3}$ ", alors la dernière et divine violence que Dieu exerce sur lui serait en fait, pour Benjamin, une violence purement révolutionnaire commise précisément sur ce premier révolutionnaire. Le passage du titre du texte dans le sous-titre, et inversement du sous-titre dans le titre, indique le "malentendu " lié à la compréhension de la révolution chez Benjamin, car celui qui exerce la violence révolutionnaire n'est jamais à la place à laquelle nous nous attendons constamment qu'il soit. Est-ce précisément cette attente trompée qui nous renvoie sans cesse à la Critique de la violence de Benjamin ? Mais, avant tout, à quoi nous attendons-nous? Nous attendonsnous à une dernière violence de dimensions catastrophiques qui supprime toute violence future et toute attente ? Nous

1. Ernst Bloch écrit sur Coré après la Seconde Guerre mondiale, comme sur die mythische Reflexe, contre ce qui est hiérarchiquement au-dessus (oben). E. Bloch, L'athéisme dans le christianisme, Paris, Gallimard, 1979, p. 98; Gesamtausgabe, Band 14, Frankfurt am Main, Suhrkamp, 1959-1978, S. 108-109.

2. Ici, je suis à nouveau le Bloch du grand livre d'après-guerre Das Prinzip Hoffnung quand il écrit sur la rébellion comme idée messianique par excellence.

3. "First left oppositionist in the history of radical politics ". M. Walzer, De l'Exode à la liberté, Paris, Calmann-Levy, 1986, p. 133 ; Exodus and Revolution, New York, Basic Books, 1985, p. 111. 
attendons-nous au sujet de cette violence positive ? Au noble sujet de la révolution? Nous attendons-nous à la justice ?

Voici maintenant ce célèbre passage de Benjamin sur la distinction entre la violence mythique et la violence divine distinction qui est le point culminant de son texte. Ce fragment est immédiatement précédé par quelques phrases ambiguës dans lesquelles Benjamin, en se réclamant de Hermann Cohen, traite de la rébellion comme caractéristique principale de la lutte contre l'esprit des législations mythiques (diminuant par là l'importance de la figure du rebelle), et de notre tâche vraisemblablement la plus importante. Le rôle nuisible que joue dans l'histoire la manifestation immédiate de la violence au niveau du mythe - c'est-à-dire, pour Benjamin, les violences juridiques (Rechtsgewalt) - exige sa suppression (derenVernichtung damit zur Aufgabe wird) : "Mais voici qu'en dernière analyse cette tâche même nous pose une fois de plus la question d'une violence pure et immédiate [einer reinen unmittelbaren Gewalt], seule capable d'exorciser le mythe. Comme, sur tous les plans, Dieu est l'anti-thèse du mythe, ainsi à la violence mythique s'oppose la violence divine, qui s'en distingue de façon radicale. La violence mythique institue le droit [mythische Gewalt rechtsetzend], la violence divine, abolit ce droit [die göttliche rechtsvernichtend] ; la première fixe des frontières, la seconde ne cesse de les supprimer ; la violence du mythe impose la faute et la malédiction, la violence divine [göttliche] réconcilie le maudit; celle-là menace, celle-ci frappe; l'une est sanglante, c'est sans verser le sang que l'autre fait mourir [so diese auf unblutige Weise letal]. À la légende de Niobé, on peut opposer le châtiment de Coré et de sa bande [Gewalt Gottes Gericht an der Rotte Korah gegenübertreten]. Il atteint des privilégiés, des Lévites [Es trifft Bevorrechtete, Leviten] ; frappant sans avertir et sans menacer, il n'hésite pas à les anéantir [trifft sie unangekündikt, ohne Drohung, schlagend und macht nicht Halt vor der Vernichtung]. Mais il possède en même temps une fonction expiatrice qui est liée de façon incontestable au caractère non-sanglant de la peine. Car le sang symbolise le simple fait de vivre [das Symbol des blossen Lebens]. Or, il faut dire, encore qu'on ne puisse ici le montrer de façon plus précise, que la suspension du pouvoir 
juridique [Auslösung der Rechtsgewalt] rétablit la culpabilité de cette simple vie naturelle; le vivant sans doute n'est que l'innocente victime d'une infortune, mais il est coupable de vivre et c'est à ce titre qu'il lui faut "expier"; la malédiction cependant ne le délivre pas d'une faute, mais bien du droit. Car ce qui cesse avec la simple vie est la souveraineté même du droit sur le vivant [die Herrschaft des Rechtes über den Lebendigen auf]. La violence mythique est une violence sanglante, qui s'exerce pour elle-même contre le simple fait de vivre, la violence divine est une violence pure, qui ne touche à la vie qu'en vue du vivant [die göttliche reine Gewalt über alles Leben um des Lebendigen willen]. L'une exige le sacrifice, l'autre l'agrée [Die erste fordert Opfer, die zweite nimmt sie an] '."

Par conséquent, Benjamin conçoit la «violence divine» comme une puissance que Dieu possède ou comme une violence qu'il exerce quand il juge Coré et sa bande. C'est son unique exemple. Ce syntagme de "violence divine" (imprécis mais courant dans les textes théologiques) a chez Benjamin quelques formulations alternatives, au moyen desquelles il s'oppose dans son texte à la violence juridique, c'est-à-dire à la violence qui crée et conserve le droit. Chez lui, la "violence divine" est à la fois la "violence pure et immédiate ", la "grève prolétarienne" de Sorel qui n'est pas violente bien qu'elle anéantisse, ainsi que la "violence pédagogique» (erzieherische Gewalt) qui est également hors du droit. La "violence divine " qui, selon Benjamin, survint une fois jadis, de même que la crise des structures juridiques de caractère mythique, fondent ensemble une nouvelle époque historique (ein neues geschichtliches Zeitalter). Benjamin annonce d'abord la venue de quelque chose de "nouveau " qui n'est plus si loin de nous que ça (ce n'est, quoi qu'il en soit, pas un nouveau droit ${ }^{2}$ ). Il affirme ensuite que la "violence révolutionnaire " n'est

1. W. Benjamin, "Prolégomènes à une critique de la violence ", Euvres choisies, Paris, René Julliard, 1959, p. 41-42 ; "Zur Kritik der Gewalt", Gesammelte Schriften, Band II-1, Frankfurt am Main, Suhrkamp, 1991, S. 199-200. J'ai modifié en plusieurs endroits la traduction de Maurice de Gandillac. Le traducteur écrit une note sur le mot "menacer " et mentionne qu'" En réalité, d'après le texte biblique, [...] Yahvé avait solennellement averti [...] du moins Moïse et Aaron".

2. Franz Rosenzweig emploie l'expression de "nouveau droit" dans un passage du 
plus impossible ; enfin, il annonce une violence complètement neuve et mystérieuse dont la venue est imminente et qui semble avoir toutes les caractéristiques du messianique et du souverain ${ }^{\mathrm{I}}$. La "violence divine" semble constamment présente car elle peut advenir dans toute forme imaginable (guerre, exécution de châtiments mortels, etc.) et inimaginable. À la fin de son texte, Benjamin insiste sur le fait que cette violence nous reste complètement obscure et incompréhensible. Il est connu que ce long passage que je viens de citer est la partie la plus importante et peut-être la plus originale de la Critique de la violence. Benjamin cherche à y formuler son argument en distinguant un genre de violence par laquelle seront jugés Niobé et Coré. La distinction lui sert à désigner et à " institutionnaliser " un nouveau genre de violence, puis à s'opposer avec force au pacifisme révolutionnaire et radical, ainsi qu'à la judéité de Kurt Hiller et à sa compréhension de la vie ${ }^{2}$. Ce passage est particulièrement compliqué car Benjamin y relit et y discute tous les éléments de son texte à la lumière de cette distinction et de la nouvelle violence repérée dans le cas de Coré.

Sans m'y arrêter trop longtemps, je souhaiterais énumérer ces éléments et éventuellement suggérer quelques sources et raisons pouvant expliquer que Benjamin introduise le cas de Coré dans son texte. Dans l'hypothèse que les "traces " qui concernent Coré et sa bande ont peut-être été précautionneusement effacées et/ou demeurent toujours encore inaccessibles à tous, mon intention est de trouver la

livre Der Stern der Erlösung ("La violence dans l'État 》) qui a été publié la même année que le texte de Benjamin. L'Étoile de la Rédemption, Paris, Seuil, 1982, p. 393 ; Der Stern der Erlösung (1921), Frankfurt am Main, Suhrkamp, 1996, S. 370.

1. «Die göttliche Gewalt, welche Insignium und Siegel, niemals Mittel heiligerVollstreckung ist, mag die waltende heißen ". "Zur Kritik der Gewalt ", S. 201.

2. Le texte de Hiller "Anti-Kain. Ein Nachwort zu dem Vorhergehenden " que Benjamin cite à partir de la revue Das Ziel (1919) est précédé par un court texte de Rudolf Leonhard "Endkampf der Waffengegner!" consacré à la grève des Spartakistes. Celui-ci s'achève par un appel à la lutte contre les armes (Kampf gegen die Waffe!, S. 23). Le texte de Hiller attaque le bolchevisme au nom d'une révolution sans arme et sans terreur : il est préférable de rester esclave plutôt que de provoquer une rébellion armée (gewalttätige Rotte), affirme Hiller à la page 27. 
justification du fait que quasiment aucun lecteur du texte de Benjamin ne s'est occupé de cette analogie ${ }^{\mathrm{I}}$.Ces " éléments ", ce sont en réalité les textes que Benjamin utilise au cours de la composition de son texte. Il est possible d'en reconstruire relativement facilement notre lecture en partant des nombreux textes auxquels nous renvoie ou ne nous renvoie pas le passage cité, ainsi que l'ensemble de la Critique de la violence, c'est-àdire en partant des sources de Benjamin.

1. Dans le premier groupe se trouvent les textes que Benjamin cite explicitement : Kant, Unger, Sorel, Cohen ${ }^{2}$ et Hiller.

2. Suit le groupe de livres, de textes ou de concepts pour lesquels on sait infailliblement qu'ils ont influencé d'une manière ou d'une autre le travail d'écriture de Benjamin. D'abord, le livre de Hugo Ball, Zur Kritik der deutschen Intelligenz (1919) : au-delà de l'emploi identique du mot de "critique ", il me semble que Benjamin s'est bien souvenu des analyses que Ball fait du livre de Dante, De Monarchia, chap. 1.1. Ensuite, évidemment, les deux livres d'Ernst Bloch, Geist der Utopie (1918) et Thomas Münzer, als Theologe der Revolution (1921). Enfin Baudelaire, que Benjamin cite et traduit " avant » ce texte : les concepts de "frappe ", de " $\operatorname{choc}^{3}$ " ou de "catastrophe ${ }^{4}$ "

1. Kurt Anglet mentionne à un endroit Coré et sa rébellion dans le livre Messianität und Geschichte. Walter Benjamins Konstruktion der historischen Dialektik und deren Aufhebung ins Eschatologische durch Erik Peterson, Berlin, Akademie Verlag, 1995, S. 35. C'est également le cas de Jacques Derrida, en un seul endroit, dans le Postscriptum du livre Force de loi (Paris, Galilée, 1994, p. 145).

2. Je rappelle deux textes hors pair de Günther Figal qui considèrent le problème de la pure volonté et d'un pur moyen sur l'exemple de l'influence de Kant et d'Hermann Cohen sur Walter Benjamin: "Recht und Moral als Handlungsspielräume", Zeitschrift für Philosophische Forschung, 1982, n. 36, S. 361-377; et "Die Ethik Walter Benjamins als Philosophie der reinen Mittel ", G. Figal, H. Folkers : Zur Theorie der Gewalt und Gewaltlosigkeit bei Walter Benjamin, Heidelberg, Texte und Materialien der FEST, 1979, S. 1-24.

3. En 1964, dans une réplique aux textes de jeunesse de Benjamin, Marcuse écrit sur le choc. W. Benjamin, Zur Kritik der Gewalt und andere Aufsätze, Frankfurt am Main, Suhrkamp, 1965, S. 105.

4. La "catastrophe" est bien sûr l'obsession de Scholem, mais elle est présente aussi chez Erich Unger au tout début du texte "Politik und Metaphysik " (" [...] jede unkatastrophale Politik ist unmetaphysisch nicht möglich "). Cf. Politik und Metaphysik, Würzburg, Königshausen \& Neumann, 1989 (1921), S. 7 (3). 
sont très fréquents chez Benjamin.

3. Un groupe de textes qui n'est quasiment jamais mentionné, mais qui a définitivement une trace significative dans la construction du texte de Benjamin, est constitué du livre de Rickert, Die Philosophie des Lebens (1920) ${ }^{\text {I }}$, de la brochure de David Baumgardt de la même année sur le problème et le concept du possible ${ }^{2}$ (et de l'impossible), et bien sûr des textes de Hegel sans lesquels le texte de Benjamin n'aurait jamais pu exister. Il ne s'agit pas seulement de la reprise par Benjamin de figures hégéliennes de la violence comme, par exemple, la violence du héros ou la "violence pure " ${ }^{3}$; il ne s'agit pas non plus seulement de la réécriture et de la correction de différents syntagmes hégéliens ${ }^{4}$; il s'agit surtout de la thématisation benjaminienne du rapport entre droit et violence qui est complètement reprise de Hegel, "un mystique de la violence " (eines Mystikers der Gewalt) 5.

4. Les textes de juristes et les textes juridiques présentent une source spécifique de l'inspiration de Benjamin. Nous n'avons aucune raison de présupposer que Benjamin ne connaissait pas les travaux de Stammler sur la théorie de l'anarchisme, sur le droit du plus fort (das Recht des Stärkeren),

1. H. Rickert, Die Philosophie des Lebens, Darstellung und Kritik der philosophischen Modeströmungen unserer Zeit, Tübingen, J. C. B. Mohr, $1920 .$.

2. Dr. D. Baumgardt, Das Möglichkeitsproblem der Kritik der reinen Vernunft, der modernen Phänomenologie und der Gegenstandstheorie, Berlin, Reuther \& Reichard, 1920. Ce livre a été publié comme "Ergänzungshefte " dans la revue Kant-Studien $\mathrm{n}^{\circ} 51$. Il serait très important pour une théorie imaginaire du possible et de l'impossible qui comprendrait Faust, Hartmann, et le Jacques Derrida tardif.

3. Die reine Gewalt. Cf. G. W. F. Hegel, Des manières de traiter scientifiquement du droit naturel, Paris, Vrin, 1972, p. 48-49 ; Fenaer Schriften. 1801-1807, Band 2, Hamburg, Felix Meiner, 1970, S. 474-475.

4. Nous retrouvons par exemple la prodigieuse distinction des violences chez Benjamin ("L'une exige le sacrifice, l'autre l'agrée " [Die erste fordert Opfer, die zweite nimmt sie an]) dans l'appendice au $\ 70$ : "Sil'État exige le sacrifice de la vie, l'individu doit y consentir. Mais l'homme a-t-il le droit de s'ôter lui-même la vie ?" Principes de la philosophie du droit, Paris, PUF, 1998, p. 157 ; Grundlinien der Philosophie des Rechts, Frankfurt am Main, Suhrkamp, Band. 7, 1970, S. 152.

5. Lettre à Scholem du 31 janvier 1918. W. Benjamin, Correspondance 19101928, t. 1, Paris, Aubier Montaigne, 1979, p. 158; Briefe I, Frankfurt am Main, Suhrkamp, 1978, S. 171. 
ou encore la série d'études de valeurs inégales imprimées à partir de 1909 en français et qui envisagent le rapport du droit à la force (et dont les auteurs sont entre autres Daniel Lesueur, Edgar Milhaud, Jacques Flach et Raoul Anthony). En revanche, il est sûr que Benjamin n'a pas pu lire avant d'avoir écrit son propre texte le livre le plus systématique qui traite du même thème, car celui-ci a été publié la même année que la Critique de la violence. Il s'agit du livre d'Erich Brodmann, Recht und Gewalt ${ }^{\text {I }}$. Cependant, pour Benjamin, le motif direct de l'écriture de son texte pourrait bien être le texte du juriste Herbert Vorwerk, "Das Recht zur Gewaltanwendung ", publié en septembre $1920^{2}$. Je ferai la supposition que ce texte et l'orageuse polémique qu'il a suscitée ont incité Benjamin à rédiger à la hâte un court texte en réponse aux problèmes du droit et de la légitimité de l'usage de la violence. La note de Benjamin au sujet du texte de Vorwerk pourrait ainsi être la première esquisse de la Critique de la violence. En même temps, la Critique constituerait vraisemblablement un résumé complet de quelques textes oubliés de Benjamin, esquisses et projets sur la politique qu'il écrivait à ce moment-là. S'il fallait maintenant que je cherche un lien entre ces trois mains (celle de Vorwerk et les deux de Benjamin, qui écrit une note puis un texte à quelques mois d'intervalle), je choisirais alors dans le texte de Vorwerk le moment qui met Benjamin à distance du droit et de la violence du droit (ou de la violence de l'État). À la page 15, Vorwerk écrit : " "Un droit à la révolution”, tel que l'enseignaient les juristes il y a déjà une centaine d'années, est conceptuellement impossible." ("Ein Recht auf Revolution ", wie es noch die Staatsrechtslehrer vor hundert fahren lehrten, ist begrifflich unmöglich).

Il n'existe pas de tel concept. Autrement dit, il n'existe pas de droit qui mène à la révolution : une révolution qui survient dans le cadre de la loi est impossible. Le syntagme de "droit

1. E. Brodmann, Recht und Gewalt, Berlin und Leipzig, Walter de Grunter \& Co., 1921.

2. À la demande du rédacteur en chef de la revue Blätter für religiösen Sozialismus, Carl Mennicke, et de son ami Paul Tillich, Vorwerk a publié ce texte dans le nº 4 (1920). 
à la révolution " est donc une pure et simple absurdité. Et à la toute fin du texte Critique de la violence, tout se passe comme si Benjamin découvrait un autre espace pour la violence et pour la révolution : "Mais si la violence, sous sa forme pure et de façon immédiate, peut s'assurer un statut durable, en deçà même $d u$ droit [jenseits des Rechtes], la preuve alors sera faite qu'une violence révolutionnaire est possible [die revolutionäre Gewalt möglich ist] " ".

Pour qu'une telle réponse soit possible, pour que ce qui est conceptuellement impossible devienne possible, il est nécessaire de changer de registre et de séparer absolument le droit et la violence. Seule la violence qui peut s'abstraire complètement du droit peut s'appeler violence révolutionnaire (divine, absolue, pure, souveraine, etc.). Cette stricte distinction est bien la condition pour la découverte d'un espace (et d'un temps) tout à fait nouveau, hors du droit. Dans la note et première réaction au texte de Vorwerk - donc quelques mois avant la Critique -, Benjamin s'oppose à la violence du droit, c'est-à-dire à la "tendance intensive du droit à sa propre effectuation [intensive Verwirklichungstendenz des Rechts] ". Son intention est de limiter la hâte et l'impatience du droit à occuper "le monde ". Il semble que cette réserve à l'égard du droit est une introduction à quelque chose de tout à fait différent. "Du rythme violent de l'impatience [Um den gewalttätigen Rhythmus der Ungeduld], dans lequel le droit existe et trouve sa mesure temporelle, au contraire du bon (?) rythme de l'attente [Rhythmus der Erwartung] dans lequel se déroule l'événement messianique [in welchem das messianische Geschehen verläuft] ${ }^{2} "$.

Le point d'interrogation qui se situe après l'adjectif "bon " (guten) est une intervention postérieure de Benjamin et constitue le propos de cette phrase. Il ne s'agit pas seulement d'une hésitation latente ou d'un délai que s'accorde Benjamin

1. W. Benjamin, "Prolégomènes à une critique de la violence ", p. 45 ; "Zur Kritik der Gewalt ", S. 202.

2. "Le droit de recourir à la violence ", W. Benjamin, Fragments, Collège / PUF, 2001, p. 115 ; Gesammelte Schriften, Band VI, S. 104. 
dans l'attente d'un adjectif plus précis, mais aussi de cette même incertitude face à l'événement annoncé comme dernier et divin. Le point d'interrogation à la fois supprime et ne supprime pas l'horizon de prévisibilité de l'événement qui se déroule déjà maintenant ${ }^{\mathrm{I}}$ et qui, en même temps, retarde sans cesse (die Verzögerung). L'événement messianique ${ }^{2}$, comme événement qui doit interrompre (et qui interrompt) la violence $\mathrm{du}$ droit, comme dernière violence qui supprime toute violence future, définit et structure l'attente (Erwartung). Seule l'attente rendra effectif ce qui est complètement impossible.

5. La lecture que fait Benjamin du texte de Vorwerk et sa manifestation de résistance envers la violence du droit nous introduit au dernier groupe de textes et de témoignages - le groupe plus important - qui "composent" la "Critique de la violence ". C'est un "texte " infini et complexe qui retrace l'amitié de Benjamin et de Scholem, et qu'il est encore aujourd'hui impossible de reconstruire. Je ne pense ici pas tant à la difficulté de classer toutes les traces que Scholem et la "relation Benjamin-Scholem" impriment dans le texte de Benjamin ${ }^{3}$, qu'aux stratégies tout à fait secrètes et incertaines

1. Ce fragment devient plus clair au regard d'un texte non encore publié de Gershom Scholem : "Walter a dit une fois : Le royaume messianique est toujours là. Ce jugement [Einsicht] contient la plus grande vérité - mais seulement dans une sphère que, à ma connaissance, personne après les prophètes n'a atteinte " (1917). La citation est extraite du texte de Michael Löwy, "Le messianisme hétérodoxe dans l'œuvre de jeunesse de Gershom Scholem ", dans J.-C. Attias, P. Gisel et L. Kennel (éd.), Messianismes. Variations sur une figure juive, Genève, Labor et Fides, 2000.

2. Cf. G. Bensussan, "Messianisme, messianicité, messianique. Pour quoi faire, pour quoi penser?", Une histoire de l'avenir, éd. J. Benoist et F. MERLInI, Paris, Vrin, 2004, p. 26-27.

3. Au-delà des quelques lettres sans lesquelles l'analyse de la «Critique de la violence " ne peut pas commencer (la lettre la plus importante étant celle à Scholem de janvier 1921), je pense aussi au travail de Scholem sur les textes originaux et à son échange ininterrompu avec Benjamin ; à la recherche initiale de Scholem sur le messianisme apocalyptique et la catastrophe ;à son génial manuscrit "Bolchevisme " (Der Bolchevismus) qui traite de la révolution juive, du royaume messianique, du sang, de la rébellion et de la célèbre "dictature de la pauvreté " (die Diktatur der Armut), Tagebücher 1913-1917, Frankfurt am Main, Jüdischer Verlag, 1995, S. 556558 ; à ces inoubliables notes de 1915 sur la révolution : "Unser Grundzug : das ist die Revolution! Revolution überall!", Ibid, S. 81 ; aux "Thèses sur le concept de 
de Scholem à l'égard des archives. Afin de me limiter au texte de Benjamin et à la "violence divine ", je mettrai de côté quelques questions irréductibles aux cas de Scholem, Adorno ou Buber, afin de ne pas lever de doutes plus significatifs sur "l'utilisation" et "la manipulation" des archives au $\mathrm{xx}^{\mathrm{e}}$ siècle. Il me semble que la mention par Benjamin de Coré et de sa bande serait considérablement plus transparente si, par exemple, l'on "trouvait " la lettre de Scholem dont se réclame Benjamin le 4 août $1921^{\text {I }}$. Il serait beaucoup plus facile de thématiser les intentions de Benjamin si les "Carnets " et la "Correspondance" de Scholem entre 1918 et 1922 étaient accessibles au public. Il en va de même en ce qui concerne les réceptions du texte de Benjamin. Combien différent serait par exemple le texte "Critique de la violence " si nous avions devant nous l'interprétation de la plus grande penseuse de la violence du siècle dernier et amie de Benjamin, Hannah Arendt.

Trois textes ou trois expériences benjaminiennes de lecteur et de témoin de l'Allemagne d'après-guerre peuvent être à la racine de l'analogie de Benjamin présente dans le titre de mon texte :

a/ Le roman de Hermann Bahr, Die Rotte Korahs, de 1919, qui traite du destin d'un baron autrichien qui découvre par surprise qu'il est le fils et l'héritier d'un détestable Juif profiteur de guerre. L'examen que mène Bahr de l'opposition entre le sang et le milieu, c'est-à-dire entre la biologie et la culture, à l'occasion de la question raciale, puis du rapport de la loi et de l'argent, de la morale et de la corruption, ainsi que son antisémitisme hystérique et sa foi paradoxale dans la régénération des Juifs - sont des éléments qui ont en toute certitude attiré l'attention de Benjamin ${ }^{2}$.

justice" de Benjamin, publiées dans les carnets de Scholem (vraisemblablement classées sans raison valable en 1916) et à la distinction capitale entre mischpatah [mishpat], Recht et zedek [zedaka], Gerechtigkeit, Ibid, S. 401-402.

1. "Tout ce que tu m'écris sur "Critique de la violence" m'a naturellement ravi. Elle paraît ces jours-ci" (Heidelberg, 04. 08. 1921). W. Benjamin, Correspondance 19101928 , t. 1, p. 248 ; Briefe I, S. 270.

2. Le roman a été publié en 1919 chez l'éditeur S. Fisher (Berlin, Wien). 
b/ Chez Kant, qu'il lit avec beaucoup d'attachement pendant de nombreuses années, Benjamin a pu remarquer un passage clé du livre La Religion dans les limites de la simple raison. Dans la seconde édition du livre (1794), Kant apporte quelques suppléments à sa formulation habituelle : "L'homme doit sortir [herausgehen soll] pour entrer (dans un état politique et civil [um in einen politisch-bürgerlichen zu treten]) ${ }^{\mathrm{I}}$ ". Dans la deuxième phrase du paragraphe, Kant utilise un pléonasme pour renforcer l'image de l'effort: "L'homme naturel doit s'efforcer de sortir" (la première édition donnait "s'efforce" au lieu de l'infinitif "s'efforcer") (der natürliche Mensch [...] herauszukommen sich befleissigen soll). Dans la suite de la phrase, Kant montre une certaine précipitation : l'homme "doit s'efforcer de sortir aussi tôt que possible" [so bald wie möglich herauszukommen sich befleissigen soll]. Dans le paragraphe suivant, Kant affirme que l'homme ne peut pas "sortir seul" parce que ce devoir concerne le genre humain et des personnes isolées (eine Vereinigung derselben in ein Ganzes); plus précisément, ce devoir "exige l'union [Vollkommenheit], [...] un système de gens [System wohlgesinnter Menschen], [...] une totalité ${ }^{2}$ ". Ce grand devoir (Pflicht) de " sortie " qui diffère de tous les autres, suppose deux conditions que Kant annonce immédiatement après : d'une part, il exige la

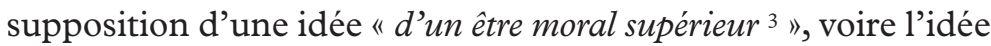
de Dieu (ce qui permettra à Kant de qualifier la communauté d'hommes parvenue à "sortir " de "peuple de Dieu "); d'autre part, il suppose une autre idée, opposée à la première et surtout à la communauté précédente : "l'idée d'une bande du principe malin " : "À un tel peuple de Dieu, on peut opposer l'idée

1. La Religion dans les limites de la simple raison, Paris, Vrin, 1972, p. 131 ; Die Religion innerhalb der Grenzen der bloßen Vernunft, Kants gesammelte Schriften, Berlin, Walter de Grunter \& Co., 1969, Band VI, S. 97.

2. Ibid, p. 132 ; Ibid, S. 98.

3. "On présume déjà par avance que ce devoir exige la supposition d'une autre idée, à savoir, celle d'un être moral supérieur [eines höhern moralischen Wesens] qui, grâce à ses dispositions générales associe les forces insuffisantes des individus [unzulänglichen Kräfte der Einzelnen] en vue d'un résultat commun. Toutefois il faut avant tout nous laisser guider d'une manière générale par le fil conducteur de ce besoin moral et voir où il nous mènera ». Ibid., p. 132 ; Ibid., S. 98. 
d'une bande $d u$ principe malin [die Idee einer Rotte des bösen Princips entgegensetzen] qui serait l'association [Vereinigung] de ceux qui sont de son parti pour répandre le mal [zur Ausbreitung des Bösen] auquel il importe de ne pas laisser se former la société opposée [jene Vereinigung nicht zu Stande kommen zu lassen] bien qu'ici encore le principe en lutte [anfechtende Princip] avec les intentions vertueuses se trouve également en nous-mêmes et ne soit représenté que figurément comme puissance extérieure [in uns selbst liegt und nur bildlich als äußere Macht vorgestellt wird] ' ."

c/ Le séminaire de Goldberg et les rencontres qu'a faites Benjamin avec des gens de son cercle (j'ai déjà évoqué plus haut Unger et Baumgardt, à qui Scholem était particulièrement allergique) pourraient éventuellement être l'aiguillon le plus important de Benjamin dans sa réflexion sur le sacrifice, le sang et la violence à propos de Coré. Pour l'instant, l'unique argument en faveur de cette hypothèse pourrait être de longs passages du livre de Goldberg paru en $1925^{2}$. Dans la rébellion de Coré (qu'il nomme Korah-Aufstand, et également KorahUnternehmen, "l'entreprise de Coré ») Oskar Goldberg voit une menace adressée au noyau métaphysique (metaphysischen Zentrums). La réaction (Reaktion) à cette rébellion - qui, selon Goldberg, est incompréhensible du point de vue "théologique " - est comme une réaction du corps (Körper) lorsqu'un de ses organes vitaux est touché (wenn ein lebenswichtiges Organ emfindlich angegriffen wird ${ }^{3}$ ).

Ces trois sources hypothétiques de l'analogie de Benjamin sont de valeurs inégales et appartiennent à des régimes textuels différents. Pourtant, si nous mettons de côté l'obscure allégorie de Hermann Bahr, on remarquera que, et chez Kant et chez Goldberg, la bande de rebelles est réduite et ramenée à une petite "partie» qui contredit le «tout». La "partie» du mal ne peut pas se constituer en entité ou en communauté qui

1. Ibid., p. 135 ; Ibid., S. 100.

2. Die Wirklichkeit der Hebräer. Einleitung in das System des Pentateuch, Erster Band, Berlin, Verlag David, 1925.

3. Ibid, S. 194-195. 
puisse subsister dans la résistance. Autrement dit, la "partie " ne peut pas subsister comme partie à l'intérieur du tout : dès lors la réaction du tout est terrible et la destruction des rebelles nécessaire. Il est intéressant d'observer que, et chez Kant et chez Goldberg, ce "principe malin " est intériorisé et présenté figurativement : comme une partie de "nous-mêmes", "en nous ", chez Kant ; comme une agression des organes de notre organisme ou de notre " corps " chez Goldberg.

Je reprends maintenant, encore une fois, le texte de Benjamin : "Comme, sur tous les plans, Dieu est l'anti-thèse du mythe, ainsi à la violence mythique s'oppose la violence divine, qui s'en distingue de façon radicale. La violence mythique institue le droit [mythische Gewalt rechtsetzend], la violence divine abolit ce droit [die göttliche rechtsvernichtend] [...]. À la légende de Niobé on peut opposer le châtiment de Coré et de sa bande [Gewalt Gottes Gericht an der Rotte Korah gegenübertreten]. Il atteint des privilégiés, des Lévites [Es trifft Bevorrechtete, Leviten]; frappant sans avertir et sans menacer, il n'hésite pas à les anéantir [trifft sie unangekündikt, ohne Drohung, schlagend und macht nicht Halt vor der Vernichtung]."

La traduction française a parfaitement dissimulé l'harmonie entre l'intervention de Benjamin et l'interprétation que font Kant et Goldberg. Il n'y a rien de "radical "; au contraire, la "violence divine " s'oppose à la violence mythique en tout point, à tous égards (in allen Stücken, in all respects); il n'y a aucun "châtiment ", bien au contraire, mais le jugement (Gericht) de Dieu préserve le tout - l'action divine ou la "violence divine " détruit et sauve en même temps (c'est pourquoi cette violence apporte la justice et non le droit ${ }^{\mathrm{I}}$ ). Contrairement à la remarque que le traducteur français, Maurice de Gandillac, fait en note ${ }^{2}$, Dieu ne donne pas d'avertissement préalable et ne menace pas ceux qu'il anéantit : il avertit seulement ceux qui l'entendent.

1. L'emploi par Benjamin des mots "procès " et "jugement " (Gericht) implique la distinction entre le droit (mishpat) et la justice (sedaqa). Dans la mesure où Dieu est le sujet d'une action qui apporte et rend la justice, son action n'est pas punitive mais protectrice. C'est là la caractéristique fondamentale de la racine $s d q$.

2. Cf. supra, n. 6. 
Mais ce n'est pas tout. Il me semble en effet que l'ambition de Benjamin va plus loin et que son emploi de Coré excède ces trois moments que j'ai distingués, et deux problèmes que j'ai évoqués et maintenus jusqu'ici au second plan. À savoir : j'ai insisté au début sur la surprise qui surgit dans le texte de Benjamin quand quelqu'un, qui semble être de gauche et être le révolutionnaire par excellence (Coré le séditieux selon Walzer) - et Benjamin parle beaucoup de la révolution dans son texte -, est subitement anéanti par la "violence divine ". J'ai ajouté plus loin un second problème qui concerne les échanges entre Benjamin et Scholem et qui suggère l'influence que ces textes sacrés et les analyses rabbiniques pourraient avoir eue sur la formation de la représentation que Benjamin se fait de Coré. Par conséquent, à la différence de Goldberg et de Kant, et en partie également de Walzer, mais aussi, à la différence beaucoup moins évidente de Scholem, Benjamin essaie de penser ensemble ce point de vue "théologique" incompréhensible (theologische Gesichtpunkte) et le geste révolutionnaire de la rébellion. C'est seulement au niveau de ce croisement de la théologie et de la révolution (et non de la politique) qu'est possible l'impossible : l'événement messianique.

Coré est le grandiose exemple du pseudo-messie et du faux révolutionnaire, mais aussi le premier initiateur du théâtre messianique et du monde à venir.

Mais pourquoi Coré est-il alors un faux révolutionnaire? La réponse la plus précise est que Coré n'est pas le Messie. Au moment où Dieu frappe et anéantit, Coré et ses compagnons, Benjamin - c'est là encore une grande surprise - les définit comme des Lévites privilégiés. Ce sont des privilégiés, Bevorrechtete ("Estrifft Bevorrechtete "; l'adjectif est bevorrechtig). Bien que ce terme peu usité montre de manière ambiguë qu'ils sont frappés et anéantis avant d'être jugés, donc avant le procès, la menace et l'avertissement, il semble que l'intention de Benjamin soit tout à fait autre. En outre, comment peuvent être dits "privilégiés " ceux qui se rebellent contre les privilèges et les "droits " à gouverner et à être prêtre, ceux de Moïse et 
d'Aaron? Comment Coré peut-il être le seul privilégié, alors même qu'il est le cousin de Moïse ? Bref, en vertu de quoi sont-ils, eux, dits privilégiés ? Benjamin n'emploie pas l'adjectif habituel privilegiert mais un mot qui contient en soi le droit, le jugement et le procès (Bevorrechtete). Par là, Benjamin se rapproche de l'interprétation "théologique" selon laquelle ils sont anéantis car ils transgressent la loi. Dieu défend la loi et anéantit tout ce qui est hors d'elle (les rebelles ou les "privilégiés "). Benjamin montre ensuite que cette rébellion n'est pas une révolution mais une création de droit ou de loi. Ils sont anéantis parce qu'ils recherchent des privilèges dans le cadre de la loi existante, et ils sont privilégiés car leur position est déjà hors du droit (ce qu'indique d'ailleurs la distinction entre leur rébellion contre Moïse et Aaron et leur rébellion contre Dieu). La raison principale pour laquelle Coré est un faux révolutionnaire privilégié tient à sa richesse et à l'influence qu'il possède au sein du peuple avant la rébellion elle-même ${ }^{\mathrm{I}}$. Il n'est pas pauvre ${ }^{2}$, c'est un homme politique, non pas un révolutionnaire. Et même si Benjamin n'avait pas connu l'origine de l'ambition de Coré (la femme de Coré joue un rôle très intéressant dans sa carrière ${ }^{3}$ ), le mot Bevorrechtete est utilisé à bon escient pour montrer qu'il est question du privilège matériel de cette bande. Par conséquent, Coré et sa bande luttent pour le pouvoir et, selon Benjamin, relèvent du domaine du droit et de la violence juridique-mythique qui n'a aucun lien avec la révolution ${ }^{4}$. Ils doivent être frappés et anéantis car

1. Cf. Sanhedrin, 110a ; Midrash Num. Rabbah, 10.3, 18.13, 22.7.

2. "Seul le jugement des pauvres a un pouvoir révolutionnaire" [Urteil des Armen hat allein revolutionäre Macht. Die Arme ist vielleicht nicht gerecht, aber er kann niemals ungerecht sein]. G. Scholem, "Der Bolschewismus ", Tagebücher 19131917, S. 556.

3. Cf. Sanhedrin, 109b - 110a ; "Il était jaloux du choix comme prince d'Elitsaphane fils d'Ouziel, que Moïse avait nommé prince... " Rachi, Le Commentaire de Bamidbar, Paris, Les éditions Biblieurope, 2005, p. 163.

4. Cf. Nombres $(16,2)$, qui commence par : "Ils se levèrent (ou ils s'avancèrent) devant Moïse ". Le syntagme "vayacoumou lifnei (Moshé) " a une connotation pleinement légale et s'emploie au tribunal pour nommer l'adversaire (Deutéronome, 19,15-16 ; Psaumes, 27,12). 
ils ne peuvent pas être constitués en communauté antagoniste (le "principe malin" de Kant), ne peuvent pas subsister comme une partie du tout ou de la communauté (cf. le différend entre Chamaï et Hillel ${ }^{\mathrm{I}}$ ) et ne peuvent jamais anéantir le droit (la loi) puisque leur intention est au contraire de le transformer en un nouveau droit (c'est-à-dire en un nouveau privilège).

Mais pourquoi, de manière générale, une telle rébellion " réformatrice " "produit " l'événement de Dieu et sa violence destructrice ? Le travail de l'analogie et de la contre-analogie - je rappelle que Benjamin, durant ces années-là, écrit un texte important sur l'analogie - aide Benjamin à tirer une conclusion conséquente: la violence absolue (destructrice, divine et révolutionnaire) anéantit la violence juridique et politique, c'està-dire la rébellion intérieure à la loi (toujours menée en vue de privilèges et de bénéfices), comme culmination de l'hypocrisie absolue du capitalisme. Inversement, la rébellion de Coré et de sa bande est en même temps la condition principale pour que cette violence nouvelle et incroyable se manifeste comme destructrice. Le faux révolutionnaire annonce le vrai révolutionnaire. Il n'y a pas de révolution sans fausse violence, sans violence mythique des rebelles ou bien sans guerre ${ }^{2}$. Ainsi, le paradigme de la pratique révolutionnaire est découvert dans l'intervention violente de Dieu, c'est-à-dire dans l'attente du Messie non-violent.

La proposition fondamentale (der Grundsatz) est celle-ci : "C'est seulement dans le monde à venir (celui de l'accomplissement) [nur in der kommenden Welt (der Erfülltheit)] que la véritable violence divine [echte göttliche Gewalt] (l'action divine immédiate [unmittelbarer göttlicher Einwirkung]) peut se manifester autrement que de manière destructrice [anders als zerstörend]. Si,

1. Dans le texte "Sitra achra; Gut und Böse in der Kabbala " (Von der mystischen Gestalt der Gottheit, Frankfurt am Main, Suhrkamp, 1973 (1962), S. 68-69 ; en français La mystique juive. Les thèmes fondamentaux, Paris, Cerf, 1986, p. 89), Scholem évoque la rébellion de Coré dans le contexte d'un grand différend entre deux docteurs : Hillel et Chamaï, cf. Le Zohar, t. I, Paris, Verdier, 1981, p. 102-106.

2. Cf. la distinction que fait Scholem entre la sanglante révolution bolchevique, l'empire messianique et la violence de la Première Guerre mondiale, Ibid. 
par contre, la violence divine fait irruption dans le monde terrestre, elle respire la destruction [atmet sie Zerstörung]. [...] Dans ce monde, la violence divine [göttliche Gewalt] est supérieure [ist höher] à la non-violence divine [göttliche Gewaltlosigkeit]. Dans le monde à venir la non-violence divine est supérieure à la violence divine. ${ }^{\mathrm{I}}$ "

Mais alors pourquoi Coré est-il un faux messie?

Il faut répondre : parce que Coré n'est pas un révolutionnaire. Bien que sa rébellion, sans le moindre doute, contienne les éléments d'une nouvelle justice à venir, bien que toutes les conditions d'un théâtre messianique soient satisfaites, Coré seul est l'archiconspirateur (archconspirator), le "déconstructeur" et le destructeur d'une communauté en exil. C'est lui qui déclenche simultanément quatre rébellions (les Lévites contre Aaron, Datan et Abiram contre Moïse, les chefs de la tribu contre Aaron, tous ensemble contre Moïse et Aaron ${ }^{2}$ ) en ayant " pris ${ }^{3}$ " avec lui et unifié deux cent cinquante enfants d'Israël.

Au début de la rébellion, Coré s'adresse à Moïse et à Aaron en ces termes $(16,3)$ : "C'est bien trop de votre part! Toute la communauté, oui, tous sont des saints [kdschim], et au milieu d'eux est le Seigneur; pourquoi donc vous érigez-vous [tinaseu] en chefs de l'assemblée du Seigneur ${ }^{4}$ ?"

De sa vie, Coré ne prononcera pas un seul mot de plus ${ }^{5}$. Il affirme ainsi que la communauté n'est pas seule sainte, mais

1.W. Benjamin, Fragments, p. 109-110 ; Gesammelte Schriften, Band VI, S. 99.

2. The fPS Torah Commentary Numbers (Bamidbar), commentaire J. Milgrom, Philadelphia - New York, The Jewish Publication Society, 5750 / 1990, p. 129. Milgrom corrige Abrabanel qui pense qu'il y a trois rébellions (p. 415).

3. "Il prit, Kora'h, fils de Ytsar, fils de Kehat, fils de Lévi...". L'emploi du passé simple du verbe "prendre ", ("il prit " [vayikach]) signifie que Coré a convaincu et rassemblé quelques-uns des guides du peuple, mais qu'il s'est également séparé de la communauté elle-même ("Il se sépara, il se sépara du reste de l'assemblée pour entretenir une dispute ", Rashi).

4. Rabbinat Français, Tel-Aviv, 1994, p. 287.

5. D'ailleurs, on n'a aucune certitude quant à la manière dont a fini Coré, s'il a été avalé comme les autres par la terre (Sanhedrin 110 a) et s'il prononce des mots qui peuvent être entendus en auscultant la voix qui provient de Gehinom (Gehenna; Sheol) : "Moïse et sa Thora sont la vérité, et nous tous sommes des mensonges" (Sanhedrin 110 b ; Baba Bathra 74 b). Cf. F. Kafka, fournal, Paris, Grasset, 1954, p. 487 (30 juillet 1917). 
que et la communauté et chaque individu (chaque partie) de cette communauté sont saints. C'est une complète nouveauté et, en même temps, une sévère calomnie. La radicalité de cette déclaration, qui remet en cause la sanctification du grand prêtre Aaron ${ }^{\mathrm{I}}$ ainsi que Moïse en tant que premier protecteur d'Aaron et intermédiaire entre le peuple et Dieu, constitue le début de nombreux malheurs. Pourtant, l'intervention (et l'intuition) de Benjamin ouvre la possibilité d'une autre interprétation de l'épisode de Coré et de son anéantissement qui, comme nous le savons, provoque une véritable catastrophe pour ce peuple, pris dans la tourmente d'un pays sauvage (outre ces 250 personnes, sont également anéantis leurs femmes et enfants, et encore 14700 compatriotes). La violence révolutionnaire de Dieu ou la "violence divine" du Messie, qui anéantit sans verser le sang, ne prononce pas seulement un "jugement" en faveur de Moïse ou d'Aaron, voire de la loi, mais indique avant tout un chemin dans le désert, et annonce le Messie nonviolent à venir et vraisemblablement une révolution tout à fait pacifique. C'est pourquoi ce terrible épisode devrait être : 1 . la mesure de toute attente à venir, ainsi que de celle de la venue du Messie (mashiah) (du messianisme) ; 2. l'indice d'un éventuel changement de statut des protagonistes et l'indice de l'acte de Dieu qui choisit, intronise et oint (mašah); 3. la mesure de tout coup $(m s ̌ h)$ à venir et de toute révolte ; 4 . la mesure de toute parole (meshiah) à venir et de tout sacrifice ; 5 . et enfin, la mesure $[$ mashahu $(m)]$ de toute mesure à venir.

Mais cela est-il vraiment possible? La distinction benjaminienne des deux violences et son appel à penser une "violence divine" dans le contexte de la rébellion de Coré sont-ils des gestes qui introduisent à la pensée d'un nouveau monde à venir ? Benjamin nous esquisse-t-il véritablement les conditions permettant de reconnaître la (dernière) violence, de s'abstenir d'être violent, d'attendre la violence, voire les conditions inconditionnelles pour le dernier acte de violence?

1. Aaron est le "prêtre/ministre du culte qui est oint " [hacohen hamoshiyach], Lev. $4: 3,5$. 
Et tout ceci, afin que la violence soit finalement anéantie, afin que l'injustice sociale soit éliminée et que la souveraineté du monde (ou d'Israël selon Maïmonide) advienne enfin?

Afin de déterminer si Dieu (ou le Messie) a commis un acte de violence, c'est-à-dire si cette violence est bien la prétendue "violence divine" et si par elle se manifestent Dieu et le monde à venir, Benjamin opère à deux niveaux d'importance égale : d'une part, en définissant les caractéristiques de cette violence paraissant complètement impossible ; d'autre part, en affirmant que cette violence est bien la violence divine par laquelle Dieu anéantit Coré. Paradoxalement, ces deux niveaux disqualifient l'idée selon laquelle Dieu n'apparaîtrait qu'à travers la violence et l'anéantissement catastrophique. De la même manière, ces deux niveaux suppriment la possibilité que des violences horribles et des guerres soient légitimées et imputées à un fictif auteur idéal. 1. À un premier niveau, Benjamin, hésitant, caractérise cette violence par différents synonymes de "violence divine ", puis considère en détail le rapport entre droit et violence. Pour que la violence commise soit imputée soit au Messie soit à Dieu, il est nécessaire que cette violence soit simultanément révolutionnaire, pure, absolue, pédagogique, mais aussi qu'elle soit sans le moindre attribut : une telle violence ne constitue ni droit ni ordre, ni n'apporte de privilège, bref, ne fait rien. Une telle violence anéantit complètement, se mesure aux victimes, bien qu'il n'y ait ni sang ni restes, "comme si rien ne s'était passé". 2. À un second niveau, Benjamin intègre cette impossible violence, cet impossible événement à l'intérieur du théâtre messianique. Le faux Messie et Coré le pseudo-révolutionnaire, lit-on, ont été avalés vifs par la terre. Cette même terre s'était déjà entrouverte pour accueillir le sang d'Abel afin de dissimuler les traces du crime de Caïn et d'en différer le jugement ${ }^{\mathrm{I}}$. Pour que la violence commise soit imputée soit au Messie soit à Dieu - ce serait vraisemblablement la conséquence de la suggestion de Benjamin -, il est nécessaire que le fait même de

1. Sanhedrin, $37 \mathrm{~b}$. 
la violence simultanément efface et conserve - protège, diffère, garde en réserve - le moment révolutionnaire et négatif d'une communauté. La suppression révolutionnaire de Coré et de sa bande exige de nouveau un redressement de la communauté, mais selon une nouvelle mesure. Cette mesure n'est possible que dans l'ombre du monde à venir, quand le Messie soulève de la terre "toute la communauté ", y compris les méchants et les rebelles ${ }^{\mathrm{I}}$. "Oui, tous sont des saints et au milieu..."

1. Sanhedrin, 108 a. 